\title{
Influencia de las estrategias cognitivas de regulación emocional en el bienestar subjetivo y psicológico en estudiantes universitarios
}

\author{
Influence of cognitive strategies of emotional regulation in the subjective \\ and psychological well-being of university students
}

\author{
Corel Mateo Canedo ${ }^{\mathrm{a},}$, María Laura Andrés ${ }^{\mathrm{a}, \mathrm{b}}$, Lorena Canet-Juric ${ }^{\mathrm{a}, \mathrm{b}}$, Josefina Rubiales ${ }^{\mathrm{a}, \mathrm{b}}$ \\ anstituto de Psicología Básica, Aplicada y Tecnología, Facultad de Psicología, \\ Universidad Nacional de Mar del Plata, Argentina \\ ${ }^{b}$ Consejo Nacional de Investigaciones Científicas y Tecnológicas (CONICET), Argentina
}

\section{Resumen}

Antecedentes: en los últimos años se ha propuesto que las estrategias cognitivas de regulación emocional (ECRE) tendrían capacidad predictiva sobre distintos indicadores de bienestar, dado que algunas de ellas serían eficientes para elevar directamente la experiencia de emociones positivas y disminuir la experiencia de emociones negativas. Objetivo: el objetivo de esta investigación fue analizar la contribución explicativa del uso de las ECRE para los niveles de bienestar subjetivo (BS) y de bienestar psicológico (BP) en estudiantes universitarios, debido a que esta población se caracteriza por una vulnerabilidad particular producto de las exigencias de la vida académica. Método: se evaluaron 84 estudiantes universitarios de la Licenciatura en Psicología de la Universidad Nacional de Mar del Plata, con una edad promedio de 23 años. Se realizaron análisis de correlación y de regresión lineal múltiple para analizar los datos. Resultados: se observó que las ECRE adaptativas (ECRE-AD) se asociaron positivamente con ambos tipos de bienestar, en tanto las ECRE no adaptativas (ECRE-NA) lo hicieron en sentido opuesto. Conclusiones: este estudio muestra la contribución explicativa del uso diferencial de las ECRE con los niveles de bienestar de estudiantes universitarios. Los resultados podrían ser un aporte al diseño de programas de promoción y prevención de la salud en esta población, dado que los sentimientos de bienestar influyen en las formas de afrontar las demandas académicas.

Palabras clave: estrategias cognitivas de regulación emocional, bienestar subjetivo, bienestar psicológico, estudiantes universitarios.

Para citar este artículo:

Canedo, C. M., Andrés, M. L., Canet-Juric, L., \& Rubiales, J. (2019). Influencia de las estrategias cognitivas de regulación emocional en el bienestar subjetivo y psicológico en estudiantes universitarios. Liberabit, 25(1), 25-40. doi: https://doi.org/10.24265/liberabit.2019. v25n1.03

\begin{abstract}
Background: in recent years it has been proposed that cognitive strategies of emotional regulation (CSER) would have predictive capacity on different indicators of well-being, since some of them would be effective to directly increase the experience of positive emotions and lessen the negative ones. Objective: this investigation aimed to analyze the explanatory contribution of using CSER to influence the levels of subjective well-being (SWB) and psychological well-being (PWB) in university students, because this population is characterized by its particular vulnerability due to the demands of academic life. Method: eighty-four (84) university students of the psychology program from the National University of Mar del Plata were evaluated, whose average age was 23 years old. Correlation and multiple linear regression analyses were conducted to assess the data. Results: adaptive CSER (CSER$\mathrm{AD}$ ) were found to be positively associated with both types of well-being, while non-adaptive CSER (CSER-NA) did so in the opposite direction. Conclusions: this study shows the explanatory contribution of the differential use of CSER in university students' well-being levels. The results could contribute to designing health promotion and prevention programs in this population, given that feelings of well-being influence the coping skills for facing the academic demands.
\end{abstract}

Keywords: cognitive strategies of emotional regulation, subjective well-being, psychological well-being, university students.

Este es un artículo Open Access bajo la licencia Creative Commons Atribución-NoComercial-CompartirIgual 4.0

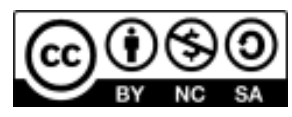




\section{Introducción}

La Psicología Positiva ha promovido que los enfoques centrados en la psicopatología se complementen con una orientación salugénica, desde donde se resalta el concepto de bienestar (Gancedo, 2008). El objetivo de los enfoques centrados en la salud es complejizar progresivamente el eje de la discusión, desde una preocupación casi exclusiva por los aspectos negativos de la vida hacia la incorporación de un conocimiento de las cualidades positivas de las personas (Seligman \& Csikzentmihalyi, 2000). La concepción de salud de esta perspectiva es amplia, considerándola un estado de bienestar físico, mental y social; y no simplemente la ausencia de enfermedad (Antonovsky, 1996).

El concepto de bienestar se concibe en términos de bienestar subjetivo (BS) y bienestar psicológico (BP). El BS ha sido definido como la evaluación cognitiva y afectiva de la propia vida. Esta percepción incluye la experiencia de emociones placenteras, bajos niveles de ánimo negativo y complacencia con la vida (Diener, Lucas, \& Oishi, 2002). Se trata de una evaluación subjetiva y global de los aspectos de satisfacción de la propia vida.

El BP refiere al grado de crecimiento total y de autorrealización a través de la resolución de desafíos evaluado como tal por la propia persona (Ryff \& Keyes, 1995). Incluye el desarrollo de la identidad, las capacidades y las relaciones positivas. Se trata de un constructo multidimensional que incluye seis componentes de funcionamiento psicológico positivo: (1) evaluación positiva de sí mismo y del pasado (autoaceptación), (2) sensación de continuo crecimiento y desarrollo como persona (crecimiento personal), (3) creencia de que la propia vida es útil y significativa (propósito en la vida), (4) mantenimiento de relaciones positivas con otros (relaciones positivas), (5) capacidad de manejar de forma efectiva la propia vida y el mundo circundante (dominio del medio ambiente) y (6) sensación de independencia y autodeterminación (autonomía) (Ryff \& Keyes, 1995). Diversos autores resaltan la importancia de su evaluación como un componente diferente pero complementario del BS, ya que aporta información valiosa sobre el funcionamiento positivo de la persona (Grant, Langan-Fox, \& Anglim, 2009; Ryan \& Deci, 2001; Ryff \& Keyes, 1995).

Niveles adecuados de BS y BP resultan predictores de una mejor calidad de vida y mayor esperanza de vida (Chanfreau et al., 2008; Steptoe, Deaton, \& Stone, 2015). Lyubomirsky, King y Diener (2005) sostienen que ambos tipos de bienestar poseen efectos positivos sobre gran número de variables relacionadas con la salud, tales como mayores niveles de sociabilidad y altruismo, fortaleza del sistema inmune, habilidades efectivas de resolución de conflictos y creatividad. Otros autores han demostrado que un mayor bienestar previene el desarrollo de psicopatologías (Suldo \& Huebner, 2004).

Dada la importancia del bienestar en la vida de los individuos, resulta de valor conocer qué factores pueden resultar predictores del mismo. Por ejemplo, para Diener (1984), la personalidad constituye un predictor consistente de BS, por ejemplo, las personas extravertidas, optimistas y sin preocupaciones suelen informar niveles más altos de felicidad. Por otro lado, ciertas estrategias de afrontamiento (creencias espirituales, valorar positivamente eventos ordinarios, reevaluación positiva y estrategias centradas en el problema) están en gran medida relacionas con el BS. En relación al BP, se ha considerado al género y la personalidad como predictores consistentes; por ejemplo, las mujeres exhiben un mayor BP que los hombres con altos puntajes en las escalas de crecimiento personal, propósito en la vida y autoaceptación (Rosa-Rodríguez, Negrón-Cartagena, Maldonado-Peña, Quiñones-Berrios, \& ToledoOsorio, 2015; Urquijo, Andrés, Valle, \& RodríguezCarvajal, 2015; Velásquez et al., 2008). A su vez, bajos niveles de neuroticismo, así como altos niveles de extraversión, apertura a la experiencia y responsabilidad constituyen importantes predictores (Augusto-Landa, Pulido-Martos, \& López-Zafra, 2010; Grant et al., 2009). Otras variables asociadas a 
ambos tipos de bienestar son las actividades religiosas, el matrimonio y la satisfacción laboral (Campbell, Converse, \& Rodgers, 1976).

Investigaciones recientes comienzan a mostrar que la regulación emocional también sería un predictor consistente de la experiencia de bienestar (e.g., Donoso, Demerouti, Garrosa-Hernández, MorenoJiménez, \& Carmona Cobo, 2015; Balzarotti, Biassoni, Villani, Prunas, \& Velotti, 2014).

La regulación emocional es definida como un conjunto de procesos intrínsecos y extrínsecos responsables de monitorear, evaluar y modificar las reacciones emocionales, especialmente su intensidad o duración, para lograr alcanzar una meta (Thompson, 1994). Las formas específicas mediante las cuales las personas regulan sus emociones son las estrategias de regulación emocional (Koole, 2009). A partir de ellas, las personas son capaces de influir en sus emociones $\mathrm{y}$ en las formas en las que se expresan.

Se han propuesto variadas formas de clasificar y agrupar las estrategias de regulación emocional. Gross, Richards y John (2006) reconocen la existencia de estrategias conscientes e inconscientes implicadas en reducir, mantener o aumentar tanto emociones positivas como negativas. Dentro de las conscientes, estas pueden estar centradas en las situaciones generadoras de emoción, en la atención o evaluación cognitiva de los estímulos y en las respuestas emocionales fisiológicas o comportamentales. Garnefski, Kraaij y Spinhoven (2001) dentro de las estrategias conscientes, se focalizan específicamente en las relacionadas con las evaluaciones cognitivas, las denominadas estrategias cognitivas de regulación emocional (ECRE) que se refieren a los pensamientos o las cogniciones que se despliegan para modularlas. Particularmente en los últimos años, las ECRE han cobrado especial relevancia teórica debido a que constituyen factores importantes en la explicación de la sintomatología clínica (Aldao \& Nolen-Hoeksema, 2010; Garnefski et al., 2001; Garnefski et al., 2002).
Garnefski et al., (2001) han seleccionado para su estudio nueve ECRE específicas: la autoculpabilización refiere a culparse a uno mismo por lo que se ha atravesado; la rumiación se refiere a pensar sobre los sentimientos o los mismos pensamientos asociados con el evento negativo; la catastrofización implica enfatizar en la gravedad de los hechos; la culpabilización de otros refiere a atribuir la culpa de la situación a los demás; la aceptación es reconocer y aceptar la realidad de lo que se ha experimentado; la refocalización positiva implica enfocarse en pensamientos alegres y positivos en vez de pensar sobre el tema que despierta emociones negativas; la planificación es adelantarse respecto de qué pasos tomar y cómo manejar el evento negativo (a pesar de que no implica necesariamente que este plan sea ejecutado); la reevaluación positiva es darle un sentido positivo a la experiencia atravesada y la toma de perspectiva refiere a relativizar la gravedad de la situación al compararla con otros eventos.

En función de las asociaciones encontradas con variables clínicas (e.g., ansiedad, depresión), las ECRE se han agrupado en adaptativas (ECRE-AD) y no adaptativas (ECRE-NA) (e.g., Garnefski \& Kraaij, 2016). Dentro de las primeras se encuentran la aceptación, la refocalización positiva, la planificación, la reevaluación positiva y la toma de perspectiva. Entre las segundas, se encuentran la autoculpabilización, la rumiación, la catastrofización y culpabilización de otros. Por ejemplo, Garnefski y Kraaij (2007) hallaron que las ECRE autoculpabilización, rumiación, catastrofización y reevaluación positiva presentaron relaciones con niveles sintomáticos de depresión y ansiedad. D'acremont y Linden (2007) encontraron relaciones entre el uso de ECRE-NA y la presencia de depresión e impulsividad, y entre el uso de ECREAD y la ausencia de este tipo de sintomatología.

$\mathrm{Al}$ evaluar el uso diferencial de ECRE en población clínica (trastornos de ansiedad y depresión) y noclínica Garnefski et al. (2002), hallaron que el primer grupo declaró una mayor frecuencia de uso de 
autoculpabilización, catastrofización, rumiación, culpabilización de otros y aceptación, mientras que el segundo grupo informó mayor uso de la reevaluación positiva. Garnefski et al. (2005) demostraron que el uso frecuente de la autoculpabilización, la rumiación y el uso poco frecuente de reevaluación positiva estaban estrechamente relacionados con el desarrollo de los denominados problemas internalizantes (tales como trastornos del estado de ánimo, ansiedad, depresión y retraimiento). Potthoff et al. (2016) observaron un uso diferencial de las ECRE en varios países europeos y hallaron que la relación de las ECRE con los síntomas psicopatológicos fue consistente a través de las distintas culturas.

En síntesis, numerosas investigaciones han analizado los correlatos del uso de las ECRE con sintomatología psicopatológica. Estos estudios aportan información valiosa para el diseño de intervenciones en problemáticas de salud mental (Garnefski et al., 2001). No obstante, se ha propuesto que las ECRE tendrían también capacidad predictiva sobre los distintos indicadores de BS y BP, debido a su rol en la elevación de la experiencia de emociones positivas y la disminución de la experiencia de emociones negativas al atravesar eventos de carácter negativo (Balzarotti et. al., 2014), por lo que resulta de valor analizar su capacidad predictiva sobre estos indicadores para una mayor y mejor comprensión de las variables relacionadas con el bienestar de las personas.

\section{Regulación emocional y bienestar subjetivo y psicológico}

El desarrollo de investigaciones respecto a la asociación específica de las ECRE con indicadores de bienestar ha sido menor (Balzarotti et al., 2014). Gross y John (2003) se centraron en el uso de una ECRE, específicamente la reevaluación cognitiva, y hallaron que las personas que utilizaban en mayor frecuencia esta estrategia experimentaban y expresaban más emociones positivas y menos emociones negativas y mostraron niveles más elevados de satisfacción con la vida, optimismo y autoestima. Haga, Kraft y Corby (2009) hallaron que el uso frecuente de reevaluación cognitiva predice niveles elevados de indicadores de bienestar (tales como afecto positivo, satisfacción con la vida y bajo estado de ánimo deprimido) en estudiantes universitarios. Balzarotti et al. (2014) hallaron que las ECRE que más se asociaron con altos niveles de BS y BP fueron la planificación y la reevaluación positiva, mientras que las estrategias de rumiación, catastrofización y autoculpabilización se mostraron vinculadas con bajos niveles de ambos tipos de bienestar. Gao, Kerstetter, Mowen y Hickerson (2018) mostraron que al utilizar estrategias cognitivas y conductuales de regulación emocional, turistas que estaban de vacaciones reportaron cambios significativos en sus niveles de bienestar.

Como puede observarse, se ha comenzado a realizar investigaciones con una orientación salugénica, buscando analizar la influencia de la regulación emocional en el funcionamiento psicológico positivo. Sin embargo, el estudio específico de la asociación entre ECRE e indicadores de bienestar es aun relativamente escaso por lo que contar con nuevos estudios que ayuden a profundizar en el conocimiento de estas relaciones resultaría un aporte complementario a lo ya estudiado.

\section{Importancia de su estudio en población universitaria}

La maleabilidad del proceso emocional, que permite a las personas la regulación de sus emociones, puede ser crucial en determinadas poblaciones y momentos vitales. Entre ellas, la población estudiantil universitaria se caracteriza por una vulnerabilidad particular debido a las exigencias que plantea la vida académica. Estas exigencias pueden provocar sentimientos negativos por no poder responder de la forma esperada (Velásquez et al., 2008). En este sentido, los estudiantes universitarios experimentan con frecuencia altos niveles de agotamiento, cinismo y falta de eficacia respecto a sus actividades académicas (Soria, Martínez, Esteve, \& Gumbau, 2005). Particularmente, los estudiantes 
de Psicología han mostrado niveles muy elevados de estrés en comparación con estudiantes de otras disciplinas (Vibe et al., 2013; Leahy et al., 2010; Thomas, Caputi, \& Wilson, 2014), produciendo efectos negativos tanto para su rendimiento académico como para su calidad de vida. Sin embargo, se ha mostrado que la autopercepción de niveles de bienestar se correlaciona con sentimientos de tranquilidad y optimismo que resultan clave para el desarrollo de estrategias frente al agotamiento y las dificultades académicas. Los sentimientos de bienestar influyen en las formas de afrontar las problemáticas cotidianas relacionadas con las demandas académicas (Velásquez et al., 2008).

Por tanto, conocer la capacidad explicativa de las ECRE en la percepción del BS y BP de estudiantes universitarios, resultaría de valor para profundizar en el conocimiento de estas relaciones y para contribuir al desarrollo de intervenciones destinadas a la promoción de la salud mental de este grupo poblacional específico. El objetivo general de esta investigación es analizar la contribución explicativa de las ECRE al BS y BP de estudiantes universitarios.

\section{Método}

\section{Diseño}

Se trata de una investigación cuantitativa con un diseño de tipo predictivo transversal. Se busca explorar la relación funcional entre el uso de las ECRE con los niveles de BS y BP de una población de estudiantes universitarios (Ato, López-García, \& Benavente, 2013)

\section{Participantes}

Se evaluaron 84 estudiantes universitarios que cursaban el tercer, cuarto y quinto año de la Licenciatura en Psicología de la Universidad Nacional de Mar del Plata. De ellos, el 85\% fueron mujeres y el $15 \%$ varones, con edad promedio de 23 años $(D E=4.41)$, seleccionados de manera no probabilística intencional.

\section{Instrumentos}

Para la evaluación de las ECRE se utilizó la adaptación de Medrano, Moretti, Ortiz y Pereno (2013) del Cuestionario de Regulación Emocional Cognitiva CERQ de Garnefksi et al. (2001). El CERQ es un instrumento de autoinforme desarrollado para investigar el afrontamiento cognitivo consciente que las personas suelen manifestar luego de la experimentación de eventos negativos. Está compuesto por nueve subescalas que representan las nueve ECRE, tanto adaptativas como desadaptativas: autoculpabilización, rumiación, catastrofización, culpabilización de otros, aceptación, refocalización positiva, planificación y toma de perspectiva. Cada subescala incluye 4 ítems cuya respuesta se mide en una escala Likert que va de 1 (casi nunca) a 5 (casi siempre). Los puntajes de las subescalas se obtienen a través de la suma de los puntajes provenientes de los ítems incluidos en cada una. El mínimo puntaje es 4 y el máximo es 20. Cuanto más alto es el puntaje en la subescala, más utilizada es la estrategia cognitiva que representa.

Los índices de consistencia interna obtenidos fueron aceptables y semejantes a los reportados en estudios antecedentes y las evidencias de validez de criterio, obtenidas al correlacionar las subescalas con medidas de emociones positivas, emociones negativas e interferencia emocional, fueron teóricamente coherentes (Medrano et al., 2013). Los valores alfa de Cronbach con sus respectivos intervalos de confianza para cada subescala en nuestra muestra bajo estudio fueron los siguientes: autoculpabilización $\alpha=.76$ (.67-.82); aceptación $\alpha=.68$ (.56-.77); rumiación $\alpha=.65$ (.53-.75); refocalización positiva $\alpha=.83$ (.76-.88); planificación $\alpha=.56$ (.41-.69); reevaluación positiva $\alpha=.72$ (.62-.80); catastrofización $\alpha=.74$ (.31-.52); toma de perspectiva $\alpha=.77$ (.69-.84); culpar a otros $\alpha=.73$ (.64-.81).

Para la evaluación del BS se administraron dos escalas. Por un lado, para la medición de la experiencia de emociones positivas y negativas, se 
utilizó la adaptación al español de Sandín et al. (1999) de la Escala de Afecto Positivo y Afecto Negativo (PANAS) de Watson, Clark y Tellegen (1988). Para la medición del componente satisfacción con la vida, se utilizó la adaptación de Atienza, Pons, Balaguer y García-Merita (2000) de la Escala de Satisfacción con la Vida (SWLS) de Diener, Emmons, Larsen y Griffin (1985).

La Escala de Afecto Positivo y Afecto Negativo (PANAS) es un cuestionario de autoinforme construido para dar cuenta de los afectos positivos y negativos que el sujeto suele sentir habitualmente. Está compuesto por 20 ítems que consisten en adjetivos que describen estados de ánimo, distribuidos en dos subescalas (afectos positivos y afectos negativos). Estos ítems se puntúan en una escala Likert que va de 1 (nada o casi nada) a 5 (muchísimo). Los puntajes de las subescalas se obtienen a través de la suma de los puntajes provenientes de los ítems incluidos en cada una. Los puntajes de afecto negativo reflejan el nivel de malestar del sujeto, mientras que los puntajes de afecto positivo reflejan el nivel de bienestar del sujeto. En cuanto a la consistencia interna del instrumento, el cálculo de los coeficientes de congruencia resultó elevado (Sandín et al., 1999). En nuestra muestra bajo estudio la subescala Afecto Positivo obtuvo un valor $\alpha=.90$ (.87-.92) y la subescala Afecto Negativo un valor $\alpha=.87$ (.77-.88).

La Escala de Satisfacción con la Vida (SWLS) es un instrumento autoadministrable que evalúa el juicio global que hacen las personas sobre la satisfacción de su vida. Incluye 5 ítems sobre la satisfacción de su vida cuya respuesta se mide en una escala Likert que va de 1 (totalmente en desacuerdo) a 5 (totalmente de acuerdo). Estos ítems son globales permitiendo a los sujetos ponderar los aspectos de su vida en base a sus propios valores para poder arribar a un juicio global de la satisfacción de su vida. El mínimo puntaje es 5 y el máximo es 25. Los niveles más altos indican una mayor satisfacción con la vida. La consistencia interna del instrumento adaptado al español mostró elevados niveles de consistencia interna mediante valores elevados de alfa de Cronbrach y correlación test-retest (Atienza et al., 2000). El valor para nuestra muestra fue de un $\alpha=.83$ (.77-.88).

Para la evaluación del BP se administró la adaptación española de Díaz et al. (2006) de la versión propuesta por Dierendonck (2004) de las Escalas de Bienestar Psicológico de Ryff (1989). Las escalas consisten en 29 ítems que los participantes responden en un formato de respuesta con puntuaciones comprendidas entre (1) «totalmente en desacuerdo» y (6) «totalmente de acuerdo». Las dimensiones se obtienen mediante un promedio de los valores obtenidos en cada reactivo, la puntuación mínima posible 1 y la máxima posible 6 en cada dimensión (Autoaceptación, Relaciones positivas, Dominio del entorno y Propósito en la vida poseen 6 ítems cada una; Crecimiento personal, 7; y Autonomía, 8). Los valores de consistencia interna del instrumento resultaron elevados. Los valores alfa de Cronbach con sus respectivos intervalos de confianza para cada subescala en nuestra muestra bajo estudio fueron los siguientes: autoaceptación $\alpha=.82$ (.75-.87); relaciones positivas $\alpha=.74$ (.65-.81); autonomía $\alpha=.55$ (.34$.67)$; dominio del entorno $\alpha=.57$ (.42-.69); crecimiento personal $\alpha=.53$ (.34-.67); propósito en la vida $\alpha=.87$ (.82-.90).

\section{Procedimiento}

Los participantes fueron contactados en la facultad en la que cursan su carrera universitaria, se les informó de los objetivos y propósitos de la investigación y aquellos que decidieron participar dieron su consentimiento informado por escrito. En el mismo, se les explicó que se trata de una investigación sobre ECRE, BS y BP de los estudiantes universitarios, así como que su participación era voluntaria y anónima y que podían interrumpirla cuando lo desearan. La administración se realizó en un único encuentro de aproximadamente 20/30 minutos por participante en aulas del complejo universitario previamente convenidas, que se encontraran sin actividad académica durante el período 
de evaluación para garantizar la continuidad de la administración y asegurar la no interrupción y silencio. La administración se realizó en las tres franjas horarias que cubre la universidad: mañana, tarde y noche.

\section{Análisis de los resultados}

Se realizaron análisis preliminares descriptivos y de correlación para conocer la media, el desvío estándar y las puntuaciones mínimas y máximas obtenidas en la muestra bajo estudio y el grado de variación conjunta de cada ECRE con cada indicador de BS y BP. Para la puesta a prueba de las hipótesis, se utilizaron análisis de regresión lineal múltiple, ya que este tipo de análisis permite conocer el grado de asociación entre cada variable independiente con la dependiente cuando se controla el efecto de las restantes independientes introducidas en el modelo.
Se calcularon los tamaños del efecto global para cada modelo de regresión lineal múltiple y parcial para cada beta estandarizada al interior de los modelos mediante el coeficiente de Cohen $f^{2}$, donde $f^{2} \geq .02, f^{2} \geq .15$, $y f^{2} \geq .35$ representan tamaños del efecto pequeños, medianos y grandes respectivamente (Cohen, 1988).

\section{Resultados}

\section{Análisis descriptivos}

La Tabla 1 muestra las puntuaciones mínimas, máximas, medias y desviaciones estándar para cada una de las variables bajo estudio. La ECRE con mayor frecuencia de uso informada fue la planificación seguida de la reevaluación positiva, la dimensión de BS con mayor frecuencia informada fue el afecto positivo y de las dimensiones de BS fueron relaciones positivas y crecimiento personal.

Tabla 1

Estadísticos descriptivos para las variables bajo estudio

\begin{tabular}{|c|c|c|c|c|c|c|c|}
\hline & & Mín. & Máx. & $M$ & $D E$ & Asimetría & Curtosis \\
\hline \multirow[t]{9}{*}{ ECRE } & Autoculpabilización & 4.00 & 19.00 & 11.65 & 3.03 & -.03 & .667 \\
\hline & Rumiación & 7.00 & 20.00 & 13.82 & 3.06 & -.30 & .051 \\
\hline & Catastrofización & 4.00 & 14.00 & 7.80 & 2.91 & .39 & -.429 \\
\hline & Culpabilización de otros & 4.00 & 15.00 & 8.34 & 2.53 & .20 & -.210 \\
\hline & Aceptación & 4.00 & 20.00 & 12.71 & 3.12 & -.42 & .855 \\
\hline & Refocalización Positiva & 5.00 & 20.00 & 12.62 & 3.36 & -.27 & .229 \\
\hline & Planificación & 11.00 & 20.00 & 16.53 & 2.19 & -.21 & .030 \\
\hline & Reevaluación Positiva & 9.00 & 20.00 & 15.72 & 2.65 & -.56 & .359 \\
\hline & Toma Perspectiva & 5.00 & 20.00 & 14.04 & 3.36 & -.48 & .276 \\
\hline \multirow[t]{3}{*}{ BS } & Afecto Positivo & 19.00 & 52.93 & 34.90 & 723 & -.11 & .27 \\
\hline & Afecto Negativo & 10.00 & 38.00 & 18.90 & 6.19 & 1.16 & 1.53 \\
\hline & Satisfacción con la vida & 12.00 & 25.00 & 19.09 & 3.35 & -.10 & .156 \\
\hline \multirow[t]{6}{*}{ BP } & Autoaceptación & 1.75 & 6.00 & 4.76 & .84 & -1.21 & 2.94 \\
\hline & Relaciones Positivas & 3.20 & 6.00 & 5.22 & .75 & -1.05 & .61 \\
\hline & Autonomía & 2.50 & 5.83 & 4.41 & .69 & -.61 & .98 \\
\hline & Dominio del Entorno & 2.40 & 6.00 & 4.67 & .73 & -.89 & .77 \\
\hline & Crecimiento Personal & 2.67 & 6.00 & 5.05 & .82 & -.85 & .60 \\
\hline & Propósito en la vida & 1.60 & 6.21 & 4.96 & .85 & -1.61 & 4.37 \\
\hline
\end{tabular}

Nota: $B S=$ Bienestar subjetivo; $B P=$ Bienestar psicológico. 


\section{Análisis de correlación}

La Tabla 2 de correlaciones muestra que la mayoría de las ECRE poseen asociaciones con los indicadores de BS y BP. Las ECRE que mayor cantidad de asociaciones presentaron con indicadores de bienestar y que a su vez mostraron los coeficientes de correlación de mayor tamaño fueron la reevaluación positiva, la refocalización positiva y la catastrofización. En menor medida, la autoculpabilización y la planificación mostraron asociaciones con el BS y BP. Las ECRE que mostraron escasa o ninguna asociación con los indicadores de bienestar fueron culpabilización de otros, rumiación, aceptación y toma de perspectiva.

Tabla 2

Correlaciones de las ECRE con los indicadores de BS y BP

\begin{tabular}{|c|c|c|c|c|c|c|c|c|c|}
\hline & $\begin{array}{c}\text { AFEC } \\
\text { POSI }\end{array}$ & $\begin{array}{l}\text { AFEC } \\
\text { NEGA }\end{array}$ & $\begin{array}{c}\text { SAT } \\
\text { VIDA }\end{array}$ & $\begin{array}{l}\text { AUTO } \\
\text { ACEP }\end{array}$ & $\begin{array}{c}\text { RELA } \\
\text { POSI }\end{array}$ & AUTO & $\begin{array}{c}\text { DOM } \\
\text { ENTOR }\end{array}$ & $\begin{array}{l}\text { CREC } \\
\text { PERS }\end{array}$ & $\begin{array}{c}\text { PROP EN } \\
\text { LA VIDA }\end{array}$ \\
\hline AUTOC & -.20 & $.28 * *$ & -.19 & $-.23 *$ & $-.25 *$ & $-.29 * *$ & -.21 & -.20 & $-.39 * *$ \\
\hline RUM & .10 & .19 & .05 & -.00 & .13 & $-.24 *$ & -.00 & -.05 & .06 \\
\hline CATAS & -.16 & $.33 * *$ & $-.24 *$ & $-.37 * *$ & $-.34 * *$ & $-.27 *$ & $-.33 * *$ & -.17 & $-.33 * *$ \\
\hline CULP OTROS & -.07 & .18 & -.11 & -.06 & -.08 & .03 & -.05 & -.10 & .07 \\
\hline ACEP & .03 & -.12 & .08 & -.05 & .13 & -.14 & -.06 & $.27 *$ & -.01 \\
\hline REFOC POS & $.27 *$ & $-.28 * *$ & $.43^{* *}$ & $.37 * *$ & $.30 * *$ & -.20 & $.31 * *$ & $.24 *$ & $.27^{*}$ \\
\hline PLAN & .15 & -.03 & .21 & $.31 * *$ & $.25 *$ & .12 & .19 & $.21^{*}$ & $.28 * *$ \\
\hline REEV POS & .17 & $-.31^{* *}$ & $.37 * *$ & $.39 * *$ & $.35^{* *}$ & .05 & $.37^{* *}$ & $.33^{* *}$ & $.35 * *$ \\
\hline TOMA DE PER & .09 & -.18 & $.26 *$ & .16 & $.36 * *$ & -.02 & .11 & .19 & .16 \\
\hline
\end{tabular}

Nota: ${ }^{*} p<.05 ; * * p<.001$

\section{Análisis de la relación entre las ECRE y los indicadores de BS y BP: regresión lineal múltiple}

Los modelos de regresión lineal múltiple resultaron significativos en todos los casos; sin embargo, el porcentaje de varianza explicado por cada uno de ellos es relativamente bajo (entre $10 \%$ y $38 \%$ ). Los tamaños del efecto fueron en su mayoría grandes $\left(f^{2}\right.$ global entre .36 y .77) y tres modelos presentaron un tamaño pequeño -i.e., afecto positivo, afecto negativo y crecimiento personal- $\left(f^{2}\right.$ global entre .22 y .35). Las ECRE con mayor capacidad predictiva sobre los indicadores de BS y BP fueron autoculpabilización, refocalización positiva, planificación y catastrofización. A diferencia del análisis de correlación anterior, la reevaluación positiva no mostró asociaciones con los indicadores de BS y BP cuando se analizó en conjunto con el resto de las ECRE. En cuanto al análisis de los coeficientes de significación práctica para cada uno de los coeficientes beta estandarizados, los coeficientes $f^{2}$ indican efectos pequeños para la mayoría de las ECRE y un efecto mediano para la ECRE autoculpabilización ( $f^{2}$ parcial $=.29$ ). Las ECRE refocalización positiva $\mathrm{y}$ planificación mostraron tamaños cercanos al nivel mediano $\left(f^{2}\right.$ parcial $=.12$ y .14 , respectivamente $)$.

En síntesis, sólo las ECRE de refocalización positiva y planificación mostraron influencia (tamaño del efecto pequeño con valores cercanos a mediano) sobre el afecto positivo, en el sentido de que mayor frecuencia de uso autoinformada de estas estrategias se asoció con mayor experiencia de afecto positivo. La ECRE autoculpabilización presentó una influencia (tamaño del efecto moderado) sobre la dimensión propósito en la vida del bienestar psicológico, en el sentido que el menor uso de esta estrategia se asocia con un mayor sentido de propósito de vida. 
Tabla 3

Relación de las ECRE con BS: análisis de regresión lineal múltiple

\begin{tabular}{|c|c|c|c|c|c|c|}
\hline & \multicolumn{2}{|c|}{ Afecto positivo } & \multicolumn{2}{|c|}{ Afecto negativo } & \multicolumn{2}{|c|}{ Satisfacción con la vida } \\
\hline Modelo & \multicolumn{2}{|c|}{$F(9)=2.21 ; p<.05$} & \multicolumn{2}{|c|}{$F(9)=3.43 ; p<.001$} & \multicolumn{2}{|c|}{$F(9)=4.36 ; p<.001$} \\
\hline$R^{2}$ & \multicolumn{2}{|c|}{.10} & \multicolumn{2}{|c|}{.18} & \multicolumn{2}{|c|}{.23} \\
\hline Intercepto & \multicolumn{2}{|c|}{24.56} & \multicolumn{2}{|c|}{20.35} & \multicolumn{2}{|c|}{11.3} \\
\hline \multirow[t]{2}{*}{$f^{2}$ Global } & \multicolumn{2}{|c|}{.22} & \multicolumn{2}{|c|}{.22} & \multicolumn{2}{|c|}{.44} \\
\hline & $ß$ & $f^{2}$ Parcial & $\beta$ & $f^{2}$ Parcial & $ß$ & $f^{2}$ Parcial \\
\hline Autoculpabilización & $-.28 *$ & .07 & $.21 *$ & .04 & $-.21 *$ & .04 \\
\hline Rumiación & .18 & .02 & .12 & .01 & .08 & .00 \\
\hline Catastrofización & -.06 & .00 & .14 & .02 & -.07 & .00 \\
\hline Culpar a otros & -.06 & .00 & .10 & .01 & -.10 & .01 \\
\hline Aceptación & .04 & .00 & -.05 & .00 & -.01 & .00 \\
\hline Refocalización positiva & $.27 *$ & .06 & -.13 & .01 & $.34 * * *$ & .12 \\
\hline Planificación & .19 & .02 & -.05 & .00 & .17 & .14 \\
\hline Reevaluación positiva & -.04 & .00 & -.14 & .01 & .08 & .00 \\
\hline Toma de perspectiva & -.07 & .00 & -.07 & .00 & .05 & .00 \\
\hline
\end{tabular}

Nota: ${ }^{*} p<.05 ;{ }^{* *} p<.01 ;{ }^{* * *} p<.001 ; f^{2} \geq .02, f^{2} \geq .15, f^{2} \geq .35$ representan tamaños del efecto pequeños, medianos y grandes respectivamente (Cohen, 1988).

Tabla 4

Relación de las ECRE con BP: análisis de regresión lineal múltiple

\begin{tabular}{|c|c|c|c|c|c|c|c|c|c|c|c|c|}
\hline & \multicolumn{2}{|c|}{ Autoaceptación } & \multicolumn{2}{|c|}{ n $\begin{array}{c}\text { Relaciones } \\
\text { Positivas }\end{array}$} & \multicolumn{2}{|c|}{ Autonomía } & \multicolumn{2}{|c|}{$\begin{array}{c}\text { Dominio del } \\
\text { Entorno }\end{array}$} & \multicolumn{2}{|c|}{$\begin{array}{c}\text { Crecimiento } \\
\text { Personal }\end{array}$} & \multicolumn{2}{|c|}{$\begin{array}{c}\text { Propósito en } \\
\text { la Vida }\end{array}$} \\
\hline Modelo & \multicolumn{2}{|c|}{$\begin{array}{c}F(9)=6.39 \\
p<.001\end{array}$} & & $F(9)=5.79$ & \multicolumn{2}{|c|}{$F(9)=4.31$; } & \multicolumn{2}{|c|}{$F(9)=3.80$} & $F(9)=.36$ & $\begin{array}{l}.36 ; \\
001\end{array}$ & $\begin{array}{c}F(9)= \\
p<.\end{array}$ & $\begin{array}{l}7.59 \\
.001\end{array}$ \\
\hline$R^{2}$ & \multicolumn{2}{|r|}{.33} & \multicolumn{2}{|r|}{.30} & \multicolumn{2}{|c|}{.23} & \multicolumn{2}{|c|}{.20} & \multicolumn{2}{|c|}{.18} & \multicolumn{2}{|c|}{.38} \\
\hline Intercepto & \multicolumn{2}{|r|}{2.85} & \multicolumn{2}{|c|}{3.72} & \multicolumn{2}{|c|}{5.23} & \multicolumn{2}{|c|}{3.58} & \multicolumn{2}{|c|}{3.09} & \multicolumn{2}{|c|}{3.11} \\
\hline \multirow[t]{2}{*}{$f^{2}$ Global } & \multicolumn{2}{|r|}{.65} & \multicolumn{2}{|r|}{.59} & \multicolumn{2}{|c|}{.44} & \multicolumn{2}{|c|}{.38} & \multicolumn{2}{|c|}{.34} & \multicolumn{2}{|c|}{.77} \\
\hline & $ß$ & $\begin{array}{c}f^{2} \\
\text { Parcial }\end{array}$ & $ß$ & $\begin{array}{c}f^{2} \\
\text { Parcial }\end{array}$ & $ß$ & $\begin{array}{c}f^{2} \\
\text { Parcial }\end{array}$ & l & $\begin{array}{c}f^{2} \\
\text { Parcial }\end{array}$ & $ß$ & $\begin{array}{c}f^{2} \\
\text { Parcial }\end{array}$ & l & $f^{2}$ \\
\hline Autoculp. & $-.21 *$ & .05 & $-.31 * *$ & .11 & $-.21 *$ & .04 & -.17 & .03 & $-.28 * *$ & .09 & $-.47 * * *$ & k. .29 \\
\hline Rumiación & -.02 & .00 & $.20 *$ & .04 & $-.27 *$ & .07 & .01 & .00 & -.04 & .01 & .14 & .02 \\
\hline Catastrof. & $-.21 *$ & .05 & $-.25 *$ & .07 & $-.24 *$ & .06 & -.17 & .02 & -.02 & .01 & $-.20 *$ & .04 \\
\hline Culpar a otros & -.008 & .00 & -.04 & .00 & .12 & .01 & .009 & .00 & -.06 & .01 & .14 & .03 \\
\hline Aceptación & -.10 & .01 & .09 & .00 & -.04 & .00 & -.12 & .01 & $.27 * *$ & .08 & .03 & .00 \\
\hline Refocal. Positiva & $.30 * *$ & .11 & .15 & .02 & $-.30 * * *$ & .09 & $.21^{*}$ & .04 & .11 & .02 & .17 & .03 \\
\hline Planif. & $.35 * *$ & $=.11$ & .19 & .03 & $.27 *$ & .06 & .14 & .01 & $.27^{*}$ & .07 & $.31 * * *$ & $=.09$ \\
\hline Reeval. Positiva & .10 & .00 & -.008 & .00 & .05 & .00 & .24 & .03 & .10 & .01 & .12 & .01 \\
\hline Toma de Perspectiva & -.06 & .00 & .18 & .03 & .01 & .00 & -.06 & .00 & -.05 & $.01-$ & -.10 & .01 \\
\hline
\end{tabular}

Nota: ${ }^{*} p<.05 ;{ }^{* *} p<.01 ;{ }^{* * *} p<.001 ; f^{2} \geq 0.02 ; f^{2} \geq 0.15 ; f^{2} \geq 0.35$ representan tamaños del efecto pequeños, medianos y grandes, respectivamente (Cohen, 1988). 


\section{Discusión}

El objetivo de este trabajo fue conocer la capacidad predictiva de las ECRE-AD y ECRE-NA sobre el BS y el BP en estudiantes universitarios. Los resultados mostraron que las ECRE tenían capacidad predictiva sobre los indicadores de BS y de BP. Estos resultados son consistentes con los escasos estudios previos que han analizado la relación entre el uso de ECRE-AD, ECRE-NA y diversos indicadores de bienestar (e.g., Gross \& John, 2003; Balzarotti, et. al., 2014).

Las ECRE con mayor frecuencia de uso fueron las ECRE-AD, de modo similar a lo informado por otras investigaciones (e.g., Garnefski \& Kraaij 2007; Balzarotti, et. al., 2014). Específicamente, la planificación fue la ECRE declarada como la más utilizada. Esta estrategia refiere a anticipar los posibles pasos de acción para manejar un evento emocionalmente activante (Garnefski et. al., 2001). La planificación es considerada generalmente como una forma de estrategia centrada en el problema, incluyendo pensamientos acerca de qué pasos seguir y cómo es la mejor forma de enfrentarlo (Balzarotti et al, 2014). Es posible que la mayor frecuencia de uso autoinformada de esta estrategia por parte de los estudiantes universitarios guarde relación con el hecho de su similitud con estrategias de planificación general de la actividad de estudio para la consecución de sus metas académicas. Para poder desempeñarse de acuerdo a las exigencias del ámbito universitario, el estudiante debe enfocarse en el manejo de su tiempo para diversas actividades, y tomar decisiones sobre a qué tareas asignar sus esfuerzos y en qué grado de intensidad (Zimmerman \& Pons, 1986).

De manera general, las ECRE predijeron la dimensión satisfacción con la vida del BS. Estos resultados podrían interpretarse considerando que el uso de ECRE-AD se asocia con un mayor control por parte del sujeto de los eventos emocionalmente activantes, pudiendo reducir los niveles de estrés y elevar los niveles de bienestar percibidos por el sujeto (Garnefski \& Kraaij, 2007; Shiota, 2006). Por otro lado, las ECRE-NA predijeron negativamente la satisfacción con la vida. Estos resultados pueden reflejar la influencia del uso de ECRE-NA en la evaluación que hace el sujeto sobre eventos negativos enfocando su atención en la gravedad de estos, dificultando la posibilidad de considerar las dimensiones del evento relacionados con el aprendizaje y el crecimiento (Balzarotti et. al., 2014).

Únicamente las ECRE refocalización positiva y planificación mostraron influencia (tamaño del efecto pequeño) sobre el afecto positivo, en el sentido de que mayor frecuencia de uso auto-informada de estas estrategias se asoció con mayor experiencia de afecto positivo. Estos resultados pueden explicarse debido a que la refocalización positiva puede resultar, a corto plazo, una estrategia adaptativa, dado que re-direcciona la atención que estaba enfocada en información emocionalmente activante, pudiendo considerarse una forma de «desconexión» (Garnefski et. al., 2001). A su vez, si se considera que el hecho de establecer metas y planificar su consecución provee un sentido de agencia personal, así como estructura y sentido a la vida diaria, se puede comprender la influencia en los niveles de bienestar de las personas. (Diener, Suh, Lucas, \& Smith, 1999).

$\mathrm{Al}$ examinar las relaciones entre las ECRE y el BP, en términos generales, las ECRE mostraron influencia sobre todas las dimensiones del BP (exceptuando crecimiento personal cuyo tamaño del efecto fue pequeño). Específicamente, la ECRE autoculpabilización mostró una influencia con un tamaño del efecto moderado sobre la dimensión propósito en la vida del bienestar psicológico, en el sentido que el menor uso de esta estrategia se asocia con un mayor sentido de propósito de vida. La autoculpabilización refiere a culparse a uno mismo por lo que se ha atravesado y se ha observado su asociación con bajos niveles de autoaceptación, autonomía y dominio del entorno (Balzarotti, 2014). Es posible considerar que esta estrategia se vincule con sentimientos de incertidumbre sobre la situación que se está viviendo, pudiendo generar la sensación 
de que no se poseen los recursos necesarios para enfrentarla, lo que llevaría a valorar de modo negativo la identidad y capacidades de la propia persona, factores centrales del BP.

Llamativamente, la reevaluación positiva no mostró contribución explicativa significativa sobre el BS y BP. La lectura de la tabla de correlaciones muestra relaciones positivas con la mayoría de los indicadores. Cuando se analiza la capacidad predictiva de esta variable controlando el posible efecto del resto de las ECRE dejan de observarse relaciones significativas. Es posible que las ECRE-AD operen en conjunto como una forma de cambio cognitivo, enfocadas en modificar la apreciación del significado emocional de una situación (Gross, 2008; John \& Gross, 2004), por lo que al ser incluidas con otras ECRE-AD posean menor poder predictivo.

En términos generales, este estudio muestra que el uso diferencial de ECRE-AD y ECRE-NA contribuye a la explicación de los niveles de bienestar de estudiantes universitarios. El hecho de que la ECRE con mayor frecuencia de uso fueran las consideradas tradicionalmente adaptativas, destacando la planificación, podría guardar relación con las características de la población universitaria.

Dentro de las limitaciones del estudio, debe mencionarse el reducido tamaño de la muestra, que podría explicar el bajo porcentaje explicativo y tamaño del efecto de algunos de los modelos, lo que dificulta las posibilidades de generalización. Por lo que un mayor número de participantes podría ampliar los niveles de representatividad, así como aumentar la precisión en la influencia del uso de las ECRE en los niveles de BS y BP. Por otro lado, ampliar la muestra a estudiantes de otras carreras universitarias, permitiría establecer comparaciones con los estudiantes de Psicología para determinar si estas relaciones varían en función de la carrera seleccionada. Es sabido que los estudiantes de Psicología se caracterizan por valerse de sus propios pensamientos y sentimientos como recursos para afrontar los sucesos de sus vidas y por ser optimistas en cuanto al futuro observando el lado positivo de las situaciones (López, 2011), lo que puede explicar parte de las asociaciones aquí encontradas. Otros factores con capacidad de influencia sobre la relación de las ECRE con BS y BP podrían ser el género, el nivel socioeconómico y los rasgos de personalidad, que no fueron controlados en la presente investigación. Por otro lado, la implementación de instrumentos de autoinforme podría haber inducido a sesgos en las respuestas del sujeto. Los resultados pueden haber sido influidos por las posibilidades del sujeto de apreciar de forma consciente el propio uso de estrategias para regular sus emociones. En futuras investigaciones, podrían incluirse el uso combinado de instrumentos de autoinforme con medidas comportamentales. Sería de valor analizar, además, el posible efecto de otras estrategias de regulación emocional, tales como estrategias comportamentales o implícitas, lo que aportaría a una comprensión más profunda del rol de la regulación emocional en el bienestar de los individuos. Finalmente, otra de las limitaciones de nuestro trabajo refiere al tipo de diseño seleccionado, que impide establecer causalidad entre las variables o una direccionalidad única respecto a su asociación. En este sentido, individuos que experimentan mayores niveles de BP y BS podrían estar más predispuestos a confiar en ECRE-AD que aquellos individuos que experimentan menores niveles. Futuros estudios deberían examinar las direcciones de influencia entre dichas variables.

A pesar de las limitaciones mencionadas, este trabajo representa un aporte al conocimiento de las variables implicadas en el bienestar en las personas. Específicamente, conocer el rol de la regulación emocional en el bienestar de estudiantes universitarios, podría contribuir al diseño de programas de promoción y prevención de la salud mental en este tipo de población. Se ha indicado, por ejemplo, que el uso de las ECRE-NA suele deberse a un desconocimiento sobre la posibilidad de ejercer control sobre el curso de las emociones, y sobre los efectos que el uso de distintas formas de regulación poseen para la vida diaria y las relaciones interpersonales (Gross, 2008). 
En este sentido, diseñar programas de intervención que promuevan la utilización de las ECRE-AD y desalienten el uso de las ECRE-NA podría repercutir de forma directa en sus niveles de bienestar y de forma indirecta podría prepararlos mejor para enfrentar las exigencias académicas de la vida universitaria.

\section{Conflicto de intereses}

Los autores manifiestan que no existen conflictos de intereses de ningún tipo.

\section{Responsabilidad ética}

Los participantes fueron contactados en la facultad en la que cursan su carrera universitaria, se les informó de los objetivos y propósitos de la investigación y aquellos que decidieron participar dieron su consentimiento informado por escrito. Los participantes autorizaron la utilización con fines docentes y la publicación con fines científicos de los datos y de los resultados obtenidos durante la investigación, siempre que se preserve la confidencialidad de los datos de todas las personas involucradas. Los datos personales de los participantes (nombre y apellido) son resguardados para preservar su privacidad y no serán publicados en este estudio. Solo se expondrá la edad y el género de los participantes.

\section{Contribución de autoría}

CMC: Recolección de datos, análisis e interpretación de los datos, redacción de versión inicial del artículo

MLA: Diseño del estudio y análisis estadístico, revisión final del manuscrito previa a la publicación

LCJ: Diseño del estudio y revisión crítica del manuscrito (contenido)

JR: Revisión crítica del manuscrito (contenido y formato)

\section{Referencias}

Aldao, A., \& Nolen-Hoeksema, S. (2010). Specificity of cognitive emotion regulation strategies: a transdiagnostic examination. Behaviour Research and Therapy, 48(10), 974-983. doi: 10.1016/j.brat.20 10.06.002

Antonovsky, A. (1996). The salutogenic model as a theory to guide health promotion. Health promotion international, 11(1), 11-18. doi: 10.1093/heapro/ 11.1.11

Atienza, F. L., Pons, D., Balaguer, I., \& García-Merita, M. (2000). Propiedades psicométricas de la Escala de Satisfacción con la Vida en adolescentes. Psicothema, 12(2), 314-319.

Ato, M., López-García, J. J., \& Benavente, A. (2013). Un sistema de clasificación de los diseños de investigación en Psicología. Anales de Psicología, 29(3), 1038-1059. doi: 10.6018/analesps.29.3.178511

Augusto-Landa, J. M., Pulido-Martos, M., \& López-Zafra, E. (2010). Emotional Intelligence and Personality Traits as Predictors of Psychological Well-Being in Spanish Undergraduates. Social Behavior and Personality: An International Journal, 38(6), 783794. doi: 10.2224/sbp.2010.38.6.783

Balzarotti, S., Biassoni, F., Villani, D., Prunas, A., \& Velotti, P. (2014). Individual Differences in Cognitive Emotion Regulation: Implications for Subjective and Psychological Well-Being. Journal of Happiness Studies, 17(1), 125-143. doi: 10.1007/s10902-0149587-3

Campbell, A. P., Converse, P. E., \& Rodgers, W. L. (1976). The Quality of American Life: Perceptions, Evaluations, and Satisfactions. Russell Sage Foundation: New York. Recuperado de http://hdl.han dle.net/2027/mdp.39015071884634

Chanfreau, J., Lloyd, C., Byron, C., Roberts, C., Craig, R., Feo, D. de, \& McManus, S. (2008). Predicting wellbeing. NatCen-DH: Londres. doi: 10.13140/2.1.3 267.4564

Cohen, J. (1988). Statistical Power Analysis for the Behavioral Sciences. Hillsdale, NJ: Lawrence Earlbaum Associates. 
D’acremont, M., \& Linden, M. van der (2007). How is impulsivity related to depression in adolescence? Evidence from a French validation of the cognitive emotion regulation questionnaire. Journal of Adolescence, 30(2), 271-282. doi: 10.1016/j.adoles cence.2006.02.007

Diener, E. (1984). Subjective well-being. Pyschological Bulletin, 95(3), 542-575. doi: 10.1037/0033-290 9.95.3.542

Diener, E., Emmons, R. A., Larsen, R. J., \& Griffin, S. (1985). The Satisfaction With Life Scale. Journal of Personality Assessment, 49(1), 71-75. doi: 10.1207/ s15327752jpa4901_13

Diener, E., Suh, E. M., Lucas, R. E., \& Smith, H. L. (1999). Subjective Well-Being: Three Decades of Progress. Psychological Bulletin, 125(2), 276-302. doi: 10.1037/0033-2909.125.2.276

Dierendonck, D. van (2004). The construct validity of Ryff's Scales of Psychological Well-being and its extension with spiritual well-being. Personality and Individual Differences, 36(3), 629-643. doi: 10.1016/ S0191-8869(03)00122-3

Donoso, L. M. B., Demerouti, E., Garrosa-Hernández, E., Moreno-Jiménez, B., \& Carmona Cobo, I. (2015). Positive benefits of caring on nurses' motivation and well-being: A diary study about the role of emotional regulation abilities at work. International journal of nursing studies, 52(4), 804-816. doi: 10.1016/j.ijnu rstu.2015.01.002

Gancedo, M. (2008). Historia de la Psicología Positiva. Antecedentes, aportes y proyecciones. En M. M. Casullo (Ed.), Prácticas en Psicología Positiva (pp. 11-38). Buenos Aires: Lugar Editorial.

Gao, J., Kerstetter, D. L., Mowen, A. J., \& Hickerson, B. (2018). Changes in tourists' perception of well-being based on their use of emotion regulation strategies during vacation. Journal of Travel \& Tourism Marketing, 35(5), 567-582. doi: 10.1080/1054840 8.2017.1374908

Garnefski, N., Baan, N., \& Kraaij, V. (2005). Psychological distress and cognitive emotion regulation strategies among farmers who fell victim to the foot-and-mouth crisis. Personality and Individual Differences, 38(6), 1317-1327.

Garnefski, N., Kraaij, V., \& Spinhoven, P. (2001). Negative life events, cognitive emotion regulation and emotional problems. Personality and Individual Differences, 30(8), 1311-1327. doi: 10.1016/S01918869(00)00113-6

Garnefski, N., Kommer, T. van den, Kraaij, V., Teerds, J., Legerstee, J., \& Onstein, E. (2002). The relationship between cognitive emotion regulation strategies and emotional problems: comparison between a clinical and a non-clinical sample. European Journal of Personality, 16(5), 403-420. doi: 10.1002/per.458

Garnefski, N., \& Kraaij, V. (2007). The Cognitive Emotion Regulation Questionnaire: Psychometric features and prospective relationships with depression and anxiety in adults. European Journal of Psychological Assessment, 23(3), 141-149. doi: 10.1 027/1015-5759.23.3.141

Garnefski, N., \& Kraaij, V. (2016). Specificity of relations between adolescents' cognitive emotion regulation strategies and symptoms of depression and anxiety. Cognition and Emotion, 32(7), 1401-1408. doi: 10.1080/02699931.2016.1232698

Grant, S., Langan-Fox, J., \& Anglim, J. (2009). The Big Five Traits as Predictors of Subjective and Psychological Well-Being. Psychological Reports, 105(1), 205-231. doi: 10.2466/PR0.105.1.205-231

Gross, J. J., \& John, O. P. (2003). Individual differences in two emotion regulation processes: implications for affect, relationships, and well-being. Journal of Personality and Social Psychology, 85(2), 348-362. doi: 10.1037/0022-3514.85.2.348

Gross, J., Richards, J., \& John, O. (2006). Emotion regulation in everyday life. In D. K. Snyder, J. Simpson, \& J. N. Hughes (Eds.), Emotion regulation in couples and families: Pathways to dysdunction and health (pp. 13-35). Washington, DC: American Psychological Association. doi: 10.1037/11468-001

Gross, J. J. (2008). Emotion regulation. In Handbook of emotions (3rd ed., pp. 497-513). New York: The Guilford press. 
Haga, S. M., Kraft, P., \& Corby, E. K. (2009). Emotion Regulation: Antecedents and Well-Being Outcomes of Cognitive Reappraisal and Expressive Suppression in Cross-Cultural Samples. Journal of Happiness Studies, 10(3), 271-291. doi: 10.1007/s10902-0079080-3

John, O. P., \& Gross, J. J. (2004). Healthy and Unhealthy Emotion Regulation: Personality Processes, Individual Differences, and Life Span Development. Journal of Personality, 72(6), 1301-1333. doi: 10.1111/j.14676494.2004.00298.x

Koole, S. L. (2009). The psychology of emotion regulation: An integrative review. Cognition and Emotion, 23(1), 4-41. doi: 10.1080/0269993080 2619031

Leahy, C. M., Peterson, R. F., Wilson, I. G., Newbury, J. W., Tonkin, A. L., \& Turnbull, D. (2010). Distress Levels and Self-Reported Treatment Rates for Medicine, Law, Psychology and Mechanical Engineering Tertiary Students: Cross-Sectional Study. Australian \& New Zealand Journal of Psychiatry, 44(7), 608-615. doi: 10.3109/000486 71003649052

López, N. (2011). Estilos de personalidad en estudiantes que ingresan a la carrera de Psicología de la Universidad Abierta Interamericana, en Rosario, provincia de Santa Fe (Tesis de licenciatura). Recuperada de http://imgbiblio. vaneduc.edu.ar/fulltext/files/TC102415.pdf

Lyubomirsky, S., King, L., \& Diener, E. (2005). The benefits of frequent positive affect: does happiness lead to success? Psychological Bulletin, 131(6), 803855. doi: 10.1037/0033-2909.131.6.803

Medrano, L. A., Moretti, L., Ortiz, Á., \& Pereno, G. L. (2013). Validación del Cuestionario de Regulación Emocional Cognitiva en Universitarios de Córdoba, Argentina. Psykhe, 22(1), 83-96. doi: 10.7764/psych e.22.1.473

Potthoff, S., Garnefski, N., Miklósi, M., Ubbiali, A., Domínguez-Sánchez, F. J., Martins, E. C., Witthöft, M., \& Kraaij, V. (2016). Cognitive Emotion Regulation and
Psychopathology across Cultures: A Comparison between Six European Countries. Personality and Individual Differences, 98, 218-224. doi: 10.1016/ j.paid.2016.04.022

Rosa-Rodríguez, Y., Negrón-Cartagena, N., MaldonadoPeña, Y., Quiñones-Berrios, A., \& Toledo-Osorio, N. (2015). Dimensiones de bienestar psicológico y apoyo social percibido con relación al sexo y nivel de estudio en universitarios. Avances en Psicología Latinoamericana, 33(1), 31-43. doi: 10.12804/apl3 3.01.2015.03

Ryan, R. M., \& Deci, E. L. (2001). On Happiness and Human Potentials: A Review of Research on Hedonic and Eudaimonic Well-Being. Annual review of psychology, 52(1), 141-166. doi: 10.1146/annurev. psych.52.1.141

Ryff, C. D. (1989). Happiness is everything, or is it? Explorations on the meaning of psychological wellbeing. Journal of personality and social psychology, 57(6), 1069-1081. doi: 10.1037/00223514.57.6.1069

Ryff, C. D., \& Keyes, C. L. (1995). The Structure of Psychological Well-Being Revisited. Journal of Personality and Social Psychology, 69(4), 719-727. doi: 10.1037/0022-3514.69.4.719

Sandín, B., Chorot, P., Lostao, L., Joiner, T. E., Santed, M. A., \& Valiente, R. M. (1999). Escalas PANAS de Afecto Positivo y Negativo: Validación Factorial y Convergencia Transcultural. Psicothema, 11(1), 37-51.

Seligman, M. E. P., \& Csikszentmihalyi, M. (2000). Positive psychology: An introduction. American Psychologis Associationt, 55(1), 5-14. doi: 10.1037/ 0003-066X.55.1.5

Shiota, M. (2006). Silver linings and candles in the dark: Differences among positive coping strategies in predicting subjective well-being. Emotion, 6(2), 335339. doi: 10.1037/1528-3542.6.2.335

Steptoe, A., Deaton, A., \& Stone, A. A. (2015). Subjective wellbeing, health, and ageing. The Lancet, 385(9968), 640-648. doi: 10.1016/S0140-6736(13)61489-0 
Suldo, S. M., \& Huebner, E. S. (2004). Does life satisfaction moderate the effects of stressful life events on psychopathological behavior during adolescence? School Psychology Quarterly, 19(2), 93-105. doi: 10.1521/scpq.19.2.93.33313

Thomas, S. J., Caputi, P., \& Wilson, C. J. (2014). Specific attitudes which predict psychology students' intentions to seek help for psychological distress. Journal of clinical psychology, 70(3), 273-282. doi: 10.1002/ jclp.22022

Thompson, R. A. (1994). Emotion Regulation: A Theme in Search of Definition. Monographs of the Society for Research in Child Development, 59(2-3), 25-52. doi: 10.1111/j.1540-5834.1994.tb01276.x

Urquijo, S., Andrés, M. L., Valle, M. del, \& RodríguezCarvajal, R. (2015). Efecto moderador del sexo en la relación a la personalidad y el bienestar psicológico en estudiantes universitarios. Liberabit, 21(2), 329340.

Velásquez, C., Montgomery, W., Montero, V., Pomalaya, R., Dioses, A., Araki, R., \& Reynoso, D. (2008). Bienestar psicológico, asertividad y rendimiento académico en estudiantes universitarios sanmarquinos. Revista de Investigación en Psicología, 11(2), 139-152. doi: 10.15381/rinvp.v11i2.3845
Vibe, M. de, Solhaug, I., Tyssen, R., Friborg, O., Rosenvinge, J. H., Sørlie, T., \& Bjørndal, A. (2013). Mindfulness training for stress management: a randomised controlled study of medical and psychology students. BMC Medical Education, 13(1), 13-107. doi: 10.1186/1472-6920-13-107

Watson, D., Clark, L., \& Tellegen, A. (1988). Development and validation of brief measures of positive and negative affect: The PANAS scales. Journal of Personality and Social Psychology, 54(6), 1063-1070. doi: 10.1037/0022-3514.54.6. 1063

Zimmerman, B. J., \& Pons, M. M. (1986). Development of a structured interview for assessing student use of self-regulated learning strategies. American educational research journal, 23(4), 614-628. doi: 10.2307/1163093 


\section{Corel Mateo Canedo}

Universidad Nacional de Mar del Plata; Instituto de Psicología Básica, Aplicada y Tecnología, Argentina

Magister en Psicología Clínica y de la Salud. Área de trabajo: Procesos de regulación de las emociones y su relación con los niveles de bienestar en estudiantes universitarios.

ORCID: 0000-0002-0620-9257

Autora corresponsal: corelmateo@gmail.com

\section{María Laura Andrés}

Instituto de Psicología Básica Aplicada y Tecnología IPSIBAT (CONICET-UNMDP) - Consejo Nacional de Investigaciones Científicas y Tecnológicas CONICET - Facultad de Psicología, Universidad Nacional de Mar del Plata, Argentina

Doctora en Psicología. Magister en Psicología, Educación y Desarrollo con especificidad en intervención psicológica en contextos de riesgo. Magister en Psicología Cognitiva y Aprendizaje. Licenciada en Psicología. Investigadora del CONICET. Docente como Jefe de Trabajos Prácticos en la Asignatura Psicología Cognitiva, Facultad de Psicología, UNMDP. Área de Trabajo: Procesos de regulación emocional en la infancia: su relación con salud mental y desempeño académico.

ORCID: 0000-0003-4398-2043

marialauraandres@gmail.com

Lorena Canet-Juric

Instituto de Psicología Básica Aplicada y Tecnología (IPSIBAT) - Consejo Nacional de Investigaciones Científicas y Tecnológicas (CONICET) - Facultad de Psicología, Universidad Nacional de Mar del Plata, Argentina

Doctora en Psicología. Magister en Psicología Cognitiva y Aprendizaje. Licenciada en Psicología. Investigadora del CONICET. Docente Facultad de Psicología, UNMDP. Área de Trabajo: Psicología Cognitiva y Educacional.

ORCID: 0000-0003-4147-4889

lcanetjuric@gmail.com

Josefina Rubiales

Instituto de Psicología Básica Aplicada y Tecnología (IPSIBAT) - Consejo Nacional de Investigaciones Científicas y Tecnológicas (CONICET) - Facultad de Psicología, Universidad Nacional de Mar del Plata, Argentina

Doctora en Psicología. Magister en Neuropsicología. Licenciada en Psicología. Profesora Adjunta Asignatura Neuropsicología, Facultad de Psicología, UNMDP. Integrante de la Comisión Académica del Doctorado en Psicología, UNMDP. Área de Trabajo: Neuropsicología infantil. Neurociencia cognitiva y afectiva del TDAH.

ORCID: 0000-0001-6842-318X

josefinarubiales@gmail.com 